\title{
État et nature du théâtre pour les jeunes en RDA entre 1949 et 1961
}

The Nature of the Theater for a Young Audience in the GDR between 1949 and 1961

\section{Astrid Persyn}

\section{OpenEdition}

Journals

Édition électronique

URL : https://journals.openedition.org/recherchestravaux/785

DOI : 10.4000/recherchestravaux.785

ISSN : 1969-6434

\section{Éditeur}

UGA Éditions/Université Grenoble Alpes

\section{Édition imprimée}

Date de publication : 1 décembre 2015

Pagination : 97-102

ISBN : 978-2-84310-314-8

ISSN : 0151-1874

Référence électronique

Astrid Persyn, «État et nature du théâtre pour les jeunes en RDA entre 1949 et 1961 », Recherches \& Travaux [En ligne], 87 | 2015, mis en ligne le 01 janvier 2017, consulté le 29 octobre 2021. URL : http:// journals.openedition.org/recherchestravaux/785 ; DOI : https://doi.org/10.4000/recherchestravaux. 785 
Astrid Persyn

Univ. Grenoble Alpes

UR LITT\&ARTS

\section{État et nature du théâtre pour les jeunes en RDA entre I949 et I96I}

Parce qu'elle représente le nombre et l'avenir aux yeux des nations et des régimes politiques, la jeunesse est toujours perçue comme un enjeu capital. Les régimes totalitaires sont sûrement à cet égard ceux qui misent le plus sur la jeunesse, conscients qu'ils sont que le formatage éducatif est la plus efficace des propagandes. C'est pourquoi les organisations de jeunesse de masse visent à la fois à la diriger et à s'assurer de son adhésion durable et sans faille au système.

L'Allemagne de l'Est socialiste n'échappa pas à cette règle. Déjà en 1946, un an seulement après la Seconde Guerre mondiale et sous occupation soviétique, elle ouvre son premier théâtre jeune public. À la création de la RDA, le parti et le syndicat national s'assurent le contrôle de la jeunesse en fondant la Freihe Deutsche Jugend ( Jeunesse libre allemande», FDJ) et son équivalent pour les enfants, les pionniers, et ils intègrent à leur cahier des charges une nécessité d'un travail "culturel et socialiste». Ce programme comportait tout un volet consacré au théâtre, et l'on emmena ces jeunes voir des pièces spécialement écrites pour eux dans des salles jeune public. Ces pièces qui étaient censées leur donner l'envie de participer à la révolution socialiste en cours, et faire naître en eux l'amour du pays, du parti et du socialisme. Sandrine Kott, historienne spécialisée dans l'histoire allemande, l'explique :

Tous les dirigeants communistes croient en l'éducabilité des personnes, mais en RDA, cette croyance est plus marquée parce qu'elle s'enracine dans une longue tradition allemande, y compris au sein du mouvement ouvrier. C'est sans doute 
dans ce pays du bloc que le souci d'éduquer l'individu a été le plus clairement affirmé et mis en œuvrer.

Cependant, tout en RDA ne fonctionnait pas forcément comme prévu et, dans les secteurs du Théâtre pour les jeunes et de l'éducation, comme dans tous les secteurs artistiques, des moyens plus ou moins dangereux de contourner les obligations virent le jour.

Quels furent ces moyens, et jusqu'où purent-ils être poussés? Après un bref retour sur la création officielle pour la jeunesse en RDA, j'aborderai les questions de controverses et de résistance interne au système, notamment à travers l'exemple de mon grand-père, Hans Dieter Johst. Cette réflexion est principalement basée sur deux ouvrages de Sandrine Kott : Histoire de la société allemande, $\mathrm{t}$. III et Theater in der $D D R^{2}$ (en collaboration). Mais je m'appuierai aussi sur de nombreux témoignages familiaux et ceux d'anciens membres d'organisations de jeunesse en RDA.

La ville de Leipzig, l'une des plus dynamiques théâtralement en RDA, fut la première à fonder en Allemagne, en novembre 1946, un théâtre consacré à la jeunesse, le Theater der Jungen Welt ("Théâtre du Jeune Monde»). Cet établissement qui existe encore aujourd'hui fut le lieu d'écriture en résidence de près de trente pièces jeunesse. La date de sa fondation correspond plus précisément à la création de la troupe permanente qu'à l'attribution d'un lieu fixe. En effet, le bâtiment qui accueillit ce théâtre, et qui était la salle blanche de la halle des congrès du zoo de Leipzig, ne lui fut officiellement affecté qu'en I947.

Il est nécessaire de rappeler que les théâtres est-allemands fonctionnent sur un principe de troupe permanente, les "Ensemble», dont le plus connu reste évidemment le «Berliner Ensemble» de Brecht. Ces troupes se composent d'un chef dramaturge, d'un chef-metteur en scène, d'un chef-scénographe, d'artisans décorateurs, de techniciens et de comédiens, dont la distribution fonctionne souvent encore sur le principe des emplois (une comédienne affectée aux rôles de jeunes premières, un comédien à ceux de vieillards, etc.).

Il est important de comprendre l'originalité de la création du Theater der Jungen Welt : certes, avant cette date, il existait déjà des spectacles jeune public, mais jamais une troupe n'avait été créée pour se consacrer uniquement au théâtre jeune public. De plus, n'oublions pas que cette fondation eut lieu juste après-guerre dans une ville bombardée. Leipzig croule encore sous les ruines lorsqu' est ouvert le Theater der Jungen Welt. La décision de la municipalité de créer un tel établissement est évidemment éminemment politique : elle permet

I. S. Kott, "La RDA I949-1989", dans Histoire de la société allemande au XXe siècle, t. III, Paris, La Découverte, 20II, p. 64.

2. S. Kott, Theater in der DDR. 
de marquer un tournant, le passage du nazisme et du capitalisme au socialisme. Il s'agit de montrer qu'une révolution est en cours. De plus, un tel lieu devait permettre de canaliser la jeunesse et de lui inculquer des valeurs socialistes.

Cependant, à cette époque, le répertoire de théâtre jeunesse est encore assez maigre en Allemagne. La première représentation de la troupe donnée à Leipzig sera donc une adaptation du roman Emil und die Detektive d'Erich Kästner, une des œuvres de littérature jeunesse germanophone les plus connues qui berça tous les enfants et jeunes Allemands de sa première publication en 1929 à aujourd'hui.

Durant les quarante ans d'existence de la RDA, le Theater der Jungen Welt fut le premier contact au monde du spectacle vivant et de la littérature dramatique de plusieurs générations d'enfants.

Peu de temps après, en 1948, on fondait à Berlin un deuxième «ensemble jeunesse»: le Theater der Freundschaft ("Théâtre de l'Amitié»), désormais appelé Theater an der Parkaue. C'est de nos jours le plus grand théâtre jeune public allemand, mais aussi le seul d'envergure nationale. Fondé sur ordre de l'administration militaire soviétique dans une ancienne école, il a la particularité d'avoir été, dès le début, composé non seulement d'une salle de représentation mais aussi de la Maison de la jeunesse, maison centrale des pionniers, l'organisation politique de masse pour les enfants jusqu'à quatorze ans. Le rôle de cet établissement était de proposer des ateliers de création artistique pour les amateurs encadrés par des professionnels et de créer tout un accompagnement culturel des jeunes dès leur plus jeune âge. Il s'agissait aussi bien d'ateliers d'écriture que de jeu ou de création de décors et costumes.

C'est dans ce théâtre qu'eut lieu la première représentation d'un texte de théâtre pour la jeunesse d'un auteur contemporain est-allemand, Du bist der Richtige (Tu es le bon) de Gustav von Wangenheim, la toute première pièce jouée au Theater der Freundschaft en 1948.

Mais cette pièce marque également les débuts de l'écriture théâtrale jeunesse officielle. Le but des représentations étant de donner envie aux jeunes de croire au socialisme, mais aussi de l'aimer et d'y participer. Plusieurs pièces virent le jour sur un modèle semblable : des personnages jeunes accomplissant un parcours initiatique finissent par apprendre une morale, et deviennent enfin de bons citoyens, fidèles au système et profondément convaincus par ses principes. Ces textes devaient donner aux jeunes, par identification, l'envie de devenir des hommes meilleurs, de trouver leur voie dans la construction du socialisme.

Cependant, il fut bien plus ardu que prévu de rallier cette jeunesse à la cause socialiste, et plusieurs moyens d'échapper à cette propagande furent trouvés à la fois par les jeunes et par les adultes en charge de leur éducation. 
Ainsi, je citerai l'exemple de mon grand-père, Hans Dieter Johst, qui témoigne de son rôle de guide d'un groupe du FDJ. Dès 1948, il fut "désigné volontaire" pour diriger environ quatre-vingts jeunes filles et garçons de quatorze à dix-huit ans, tous apprentis dans la maison d'édition Reclam où luimême travaillait, à Leipzig.

Il devait faire l'éducation culturelle de ces jeunes pendant trois ans. Il passa tout son temps libre à les amener au théâtre, à l'opéra, ou même au cinéma. Les théâtres de Leipzig se piquèrent de curiosité pour ce groupe si avide de culture, au point qu'il reçut de très nombreuses places gratuites pour les spectacles, pour les jeunes comme pour leurs familles, et put participer à plusieurs répétitions générales ainsi qu’à des discussions sur la programmation.

Étant aussi guide de ces jeunes pour des voyages et promenades, Hans Dieter Johst organisa pour eux des voyages en Suisse allemande ou à la mer, en leur interdisant systématiquement de porter leur uniforme du FD. Il amenait souvent un gramophone et des vinyles de jazz pour faire découvrir aux jeunes gens des musiques, notamment de l'Ouest. Au sein de ce groupe, il créa un club de danse folklorique, une chorale, mais aussi une troupe de théâtre amateur pour laquelle il mettait en scène des pièces représentées à l'occasion des fêtes de la maison d'édition devant les quelque six cents travailleurs et responsables de l'entreprise Reclam.

Malheureusement, bien que ce groupe fût reconnu dans l'ensemble de l'État pour la qualité de son travail culturel, il attira l'attention du SED (Sozialistische Einheitspartei Deutschlands, ou Parti socialiste unifié allemand) : pour qui un groupe «en civil», qui faisait certes un excellent travail culturel, mais manquait à ses devoirs idéologiques et citoyens envers le parti, posait problème. Dans les hautes sphères du parti, ce désintérêt des quatre-vingts jeunes et de leur guide pour le socialisme était évident : trop de culture était "un frein pour l'engagement politique et idéologique», alors que précisément ces organisations avaient pour but assurer le soutien idéologique des jeunes générations au parti.

En juillet 1952, lors d'une assemblée chez Reclam, les dirigeants du SED exigèrent que l'on sépare Hans Dieter Johst de «ses» jeunes : trop de travail culturel, pendant trois ans, avait tenu les jeunes éloignés de tout endoctrinement socialiste! Selon le SED, il les aurait "séduits avec ses tendances de l'Ouest»; aucun des jeunes garçons n'était devenu bénévole dans l'armée populaire, aucune des jeunes filles n'était rentrée dans une organisation féminine du parti. Johst se défendit en répondant qu'après-tout, il avait été mandaté pour un travail culturel, des voyages et randonnées, et non pour un enseignement politique. Les applaudissements des jeunes à ces propos furent interprétés comme la preuve de l'influence néfaste qu'il avait eue sur eux et de leur dépendance 
vis-à-vis de lui. Devenu ainsi du jour au lendemain ennemi politique, il dut fuir, la nuit même, la RDA.

Ce témoignage montre bien que le travail culturel était certes perçu comme nécessaire par les dirigeants est-allemands, mais qu'il ne devait en aucun cas passer avant l'éducation politique, autrement dit l'enrôlement des jeunes Allemands qui restait l'enjeu principal de ces organisations de jeunesse.

Cependant, le passage de Hans Dieter Johst au sein du groupe de FDJ de Leipzig aura permis la réunion d'archives uniques, notamment les comptes rendus du club de théâtre comportant la description de toutes les représentations auxquelles avaient assisté les jeunes pendant ces trois années, ce qui est précieux.

Parmi ces documents, plusieurs sont caractéristiques de la programmation des théâtres est-allemands. Prenons par exemple l'année 1950, durant laquelle six spectacles furent programmés dans le cadre du club de théâtre pour les premiers mois de la saison théâtrale de Leipzig : Moskauer Charakter de Sofronow, un auteur russe contemporain; Faust I de Goethe; Kalkutta 4. Mai de Feuchtwanger, auteur est-allemand exilé aux USA, qui reçut le Prix national d'art et littérature de la RDA; Beaucoup de bruit pour rien de Shakespeare; Les Voleurs de Schiller, et Golden Boy de Odet, un dramaturge américain stanislavskien. Ce genre de programmation était typique : des pièces russes, des classiques du répertoire allemand, des pièces est-allemandes contemporaines et quelques pièces étrangères mais inscrites dans une esthétique proche de celle imposée en RDA. Cependant, le compte rendu de cette saison indique que «la surreprésentation de pièces soviétiques et russes a été perçue comme trop importante par rapport aux pièces allemandes", et se concluait même par "n'y a-t-il donc pas d'autres auteurs dramatiques contemporains que Wolf?».

Les membres du groupe allaient régulièrement au Theater der Jungen Welt de Leipzig, mais à propos de la programmation de la saison 1950, le compte rendu du cercle de théâtre indique : "Cinq pièces, ce n'est pas un gros programme, mais un but, pas de grands drames, mais des pièces qui apportent à la jeunesse quelque chose en dehors de l'école, qui sont facilement compréhensibles et qui sont sûrement reçues avec beaucoup d'enthousiasme." La programmation de cette salle jeune public n'était donc pas abondante, et pas forcément d'une grande qualité mais elle devait suffire à divertir et instruire les jeunes spectateurs par ses créations originales.

Du côté de la jeunesse, une autre forme d'opposition se fit jour. Malgré plusieurs réformes de l'éducation telles que l'école primaire unique mixte et gratuite pour tous en 1946, la création de dix niveaux scolaires allant de six à seize ans en 1951, le système éducatif peine à créer une égalité entre les élèves. Sandrine Kott note que "l'école tend à favoriser la reproduction sociale», et 
explique : «les enfants d'ouvriers, dont les résultats scolaires sont en moyenne plus médiocres et qui sont politiquement plus rebelles, sont chroniquement sous-représentés dans les lycées ${ }^{3}$. De plus, ce sont les notes des élèves ainsi que l'appartenance des parents ou non à des organisations politiques étatiques qui déterminent les études ou formations que pourront suivre les enfants. «Dans une grande partie des cas, le jeune se prépare donc à un métier qu'il n'a pas choisi et qui lui a été imposé par l'État planificateur ${ }^{4}$." L'école est le premier lieu de politisation de la jeunesse, de propagande et d'enrôlement, mais cette propagande finit par se heurter à une résistance des jeunes : «en dépit d'un discours persistant, les élèves continuent ainsi de nourrir une vision généralement positive de la RFA et de souhaiter l'unification'». Mais bien plus que la jeunesse en formation professionnelle, tels les jeunes du FDGB de Reclam Leipzig, ce sont les lycéens et les étudiants, qui font preuve d'une certaine opposition politique dans les premières années de la RDA; les apprentis et les ouvriers sont bien moins critiques vis-à-vis de l'enseignement et de l'État. Les premiers sont véritablement tiraillés entre la volonté d'ériger une égalité de fait entre les enfants, et une éducation qui divise les élèves en deux camps, ceux fidèles au parti, membres du FDGB et dont les parents sont impliqués dans le parti unique ou dans le syndicat national, à qui les portes des universités et des centres de loisirs sont grandes ouvertes, et les autres. C'est donc principalement à cette contradiction qu'ils s'attaquèrent plus ou moins ouvertement.

C'est néanmoins sur eux que dut s'appuyer la population est-allemande pour rester libre dans sa création et dans sa réception d'œuvres dramatiques. Mais cette fois-ci le détour n'est pas la question «comment s'adresser spécifiquement à la jeunesse?», mais bel et bien "comment faire réfléchir cette jeunesse tout en restant politiquement conforme à ce qui est attendu?». Conforme est en effet le mot juste, puisque c'est le principal moyen qui fut utilisé : garder la forme de la création officielle, en garder les codes et la langue, en garder les trames et les personnages, mais détourner certains éléments par l'apparition d'un personnage plus subversif, par un jeu plus expressif, par des discussions libres avec les jeunes après les lectures et représentations... Ces moyens restèrent limités; ils permirent cependant, comme le souligne Sandrine Kott, que cette jeunesse ne soit jamais l'argile modelable à souhait qu'avait espéré façonner le Parti à travers son système d'éducation et d'enrôlement par la culture.

3. S. Kott, Histoire de la société allemande, ouvr. cité, p. 65.

4. Ibid., p. 66.

5. Ibid., p. 68. 